\title{
Barriers to optimal screening and vaccination of hepatitis B contacts: a survey of general practitioners in NSW, Australia
}

\section{Zeina Najjara,d, Janice Pritchard-Jones ${ }^{b}$, Siaw-Teng Liaw ${ }^{c}$ and Leena Gupta $^{a}$}

a Public Health Unit, Sydney Local Health District, NSW, Australia

b AW Morrow Gastroenterology and Liver Centre, Royal Prince Alfred Hospital, Sydney, NSW, Australia

c General Practice Unit, South Western Sydney Local Health District, NSW, Australia

${ }^{d}$ Corresponding author: zeina.najjar@health.nsw.gov.au

\section{Article history}

Publication date: December 2017

Citation: Najjar Z, Pritchard-Jones J, Liaw S-T, Gupta L. Barriers to optimal screening and vaccination of hepatitis $B$ contacts: a survey of general practitioners in NSW, Australia. Public Health Res Pract. 2017;27(5):e2751749. https://doi. org/10.17061/phrp2751749

\section{Introduction}

It is estimated that at least 218000 Australians are living with chronic hepatitis $B(\mathrm{CHB})$ infection, but almost half of these remain undiagnosed, partly because of the often asymptomatic nature of the infection. ${ }^{1}$ The Australian National hepatitis B strategy 2014-2017²acknowledges the increasing public health burden of $\mathrm{CHB}$ and emphasises the management of $\mathrm{CHB}$ in the primary care setting, and the testing and vaccination of priority populations, including close contacts of known cases. Close contacts are most at risk of infection; however, a previous study demonstrated that recommended screening and vaccination of close contacts is occurring suboptimally ${ }^{3}$, despite the availability of a free hepatitis B vaccine for contacts in Australia. The Sydney and South Western Sydney Local Health Districts (S\&SWSLHDs) have the highest prevalence of CHB in New South Wales. ${ }^{4}$ We surveyed general practitioners (GPS) in the area to determine their contact management practices for $\mathrm{CHB}$ patients and any barriers they may encounter.

\section{Methods}

We surveyed GPs in S\&SWSLHDs who had at least one patient notified as having $\mathrm{CHB}$ to the public health unit (PHU) between 1 June 2012 and 31 May 2013. The methods and study limitations are detailed elsewhere. ${ }^{4}$ Here, we report only on the contact management component of the survey.

\section{Results}

Completed questionnaires were returned by 123 of 213 eligible GPs (57.7\% response rate). There were significant differences in gender, age group, and type of practice between study participants and all GPs in S\&SWSLHDs. ${ }^{4}$ The average number of patients with $\mathrm{CHB}$ that respondents had notified during 
the study period was slightly less $(n=1.88)$ than that of nonrespondents $(n=1.96)(p=0.73)$.

Of the respondents, 119 GPs (96.7\%) felt 'very' or 'reasonably' confident about screening and vaccinating close contacts of patients with $\mathrm{CHB}$, yet nearly half ( $n=56,45.5 \%$ ) of GPs needed more information about eligibility requirements for the free hepatitis $B$ vaccine.

The majority of GPs surveyed ( $n=109,88.6 \%$ ) indicated that they encountered at least one problem when trying to screen and vaccinate close contacts of their patients with $\mathrm{CHB}$, with 45 (36.6\%) encountering one difficulty, and 64 (52.0\%) encountering two or more difficulties. Table 1 lists the most common difficulties encountered.

Table 1. Difficulties encountered by study participants when trying to screen and vaccinate close contacts of patients with $\mathrm{CHB}$

\begin{tabular}{cc} 
Difficulty & $\begin{array}{c}\text { Number (\%) of } \\
\text { respondents }\end{array}$ \\
\hline
\end{tabular}

The contacts are patients of another doctor/ practice

$67(54.5)$

Tried to, but patient will not provide me with information to establish identity of contacts

Patient has not returned to receive result

Cannot get in touch with any of the contacts

The person is no longer my patient

Other

Note: More than one response was allowed.

Despite the difficulties that GPs experience, more than half of the GPs surveyed ( $n=64,52.0 \%$ ) believed that the primary responsibility for tracing of household and sexual contacts of patients with CHB should lie with the GP, with support from the PHU in difficult circumstances. A total of $38(31.0 \%)$ believed this responsibility should lie with the PHU. Only 7 GPs (5.7\%) believed it should lie solely with GPs.

\section{Discussion}

Early diagnosis of $\mathrm{CHB}$ through screening of at-risk populations, including close contacts, is important for ensuring that appropriate management is initiated early and ongoing transmission is controlled. ${ }^{3}$ We found that, although GPs working in a CHB high-prevalence area were confident with contact management and believed it was primarily their responsibility, the majority encountered difficulties when doing this. If GPs are to successfully have an increased role in preventing ongoing transmission through screening ${ }^{2}$, a system that facilitates contact management and addresses the barriers encountered is required. One of the most commonly described difficulties with contact management was the patient not providing information about contacts. Many GPs also indicated that patients had not returned to receive their results. This may be explained by $\mathrm{CHB}$ disproportionately affecting migrant populations, who are often already marginalised, have variable levels of health literacy and understanding of the severity of $\mathrm{CHB}$, and attach stigma to $\mathrm{CHB}$ infection. ${ }^{2}$ Other barriers related to a lack of continuity of care, particularly where contacts were seen by a different GP.

No national protocol exists that stipulates who is responsible for contact tracing. Active follow-up of all notifications by public health services, in conjunction with GPs, would be ideal to deal with these issues, but it is resource intensive and not necessarily feasible ${ }^{5}$; alternative methods for contact tracing therefore need to be explored. These could include passive follow-up by public health services by automatically sending letters to all cases about the need for contact screening, or the use of an electronic patient-initiated contact referral system, as used for contact tracing with sexually transmissible infections.

We identified significant barriers to optimal contact management by GPs, indicating that different approaches are required to support GPs as they take on a greater role in CHB management, and allow them to more effectively screen and vaccinate close contacts. Further research may identify whether these barriers also exist in Australian $\mathrm{CHB}$ low-prevalence areas. A national protocol for the public health response to notifications of $\mathrm{CHB}$ is currently being developed, which addresses the important role of primary care. This may address some of the contact tracing issues identified in our study.

\section{Acknowledgements}

We acknowledge Simone I Strasser, Miriam T Levy, and Benjamin C Cowie for their role in the development and analysis of this survey and its results.

\section{Letter to the Editor}

To read correspondence in response to this article, click here.

\section{Competing interests}

None declared

\section{Author contributions}

ZN was the lead author and responsible for the study design and coordination, data collection, data analysis and writing the manuscript. JP-J, S-TL and LG contributed to the study design and writing the manuscript. 


\section{References}

1. Allard N, MacLachlan J, Cowie B. The cascade of care for Australians living with chronic hepatitis B: measuring access to diagnosis, management and treatment. Aust N Z J Public Health. 2015;39(3):255-9.

2. Australian Government Department of Health. Second National Hepatitis B Strategy 2014-2017. Canberra: Commonwealth of Australia; 2014 [cited 2016 May 27]. Available from: www.health.gov.au/internet/main/ publishing.nsf/Content/C353814FE3255962CA257BF000 1DE841/\$File/Hep-B-Strategy2014-v3.pdf
3. Williams S, Vally H, Fielding J, Cowie B. Hepatitis B prevention in Victoria, Australia - the potential to protect. Euro Surveill. 2011;16(22): pii=19879.

4. Najjar Z, Gupta L, Pritchard-Jones J, Strasser S, Liaw S-T, Cowie BC. A survey of Sydney general practitioners' management of patients with chronic hepatitis B. Med J Aust. 2016;204(2):74.

5. Leder K. Public health management of hepatitis B virus contacts. Med J Aust. 2013;198(7):366.

\section{Copyright: (c) (i) (-)}

(C) 2017 Najjar et al. This article is licensed under the Creative Commons Attribution-NonCommercial-ShareAlike 4.0 International Licence, which allows others to redistribute, adapt and share this work non-commercially provided they attribute the work and any adapted version of it is distributed under the same Creative Commons licence terms. See: www.creativecommons.org/licenses/by-nc-sa/4.0/ 